\title{
CAPITULO 08
}

\section{A IMPORTÂNCIA DO TRABALHO DA EQUIPE MULTIDISCIPLINAR NO PROGRAMA SAÚDE DA FAMÍLIA}

$\underline{\text { DOI 10.4322/978-65-995353-2-1.c08 }}$

\author{
$\underline{\text { Angelica Ribeiro do Nascimento Oliveira }}{ }^{1}$, Ana Luisa Mendes Ribeiro² ${ }^{2}$ Eliana Luz \\ Lopes $^{3}$, Lara Beatriz de Sousa Araújo ${ }^{4}$, Maria Cecilia do Nascimento Fontinele ${ }^{5}$, Lívia \\ Karen Barbosa de Brito ${ }^{6}$, Rafaela de Jesus Portugal ${ }^{7}$, Sabrina Sousa Fontenele ${ }^{8}$, Ynara \\ Maria Gomes de Sousa ${ }^{9}$, Caroline Taiane Santos da Silva ${ }^{10}$ \\ 1Universidade Maurício de Nassau, Unidade Teresina, (angelicalribeiro.ar19@gmail.com) \\ 2Universidade Federal do Piauí, (analuuhm28@gmail.com) \\ 3Universidade Federal do Oeste da Bahia, (eliana.11930@ufob.edu.br) \\ ${ }^{4}$ Universidade Federal do Piauí, (larabeatriz@ufpi.edu.br) \\ ${ }^{5}$ Universidade Federal do Piauí, (mariacecilia0512@ hotmail.com) \\ ${ }^{6}$ Universidade Federal do Piauí, (liviakarenbb@gmail.com) \\ ${ }^{7}$ Universidade Salvador - UNIFACS, (rafaelaenfermagem2@gmail.com) \\ ${ }^{8}$ Universidade da Amazônia, (sabrinafontnele@gmail.com) \\ ${ }^{9}$ Universidade Federal do Piauí, (ynara@ufpi.edu.br) \\ ${ }^{10}$ Child Behavior Institute, (carolinetaiane.enfa@gmail.com)
}

\section{Resumo}

Objetivos: Identificar na literatura científica a importância do trabalho da equipe multidisciplinar no programa saúde da família. Método: Trata-se de uma revisão integrativa, utilizando para fins de coleta de dados as bases de dados e bibliotecas online, como a Scientific Electronic Library Online (SciELO) e Literatura Latino-americana e do Caribe em Ciências da Saúde (LILACS). Aplicando os descritores arrolados na plataforma Decs: Estratégia Saúde da Família; Equipe de assistência ao paciente, atenção primária à Saúde. Os descritores foram combinados entre si pelo operador booleano "AND". Foram incluídos artigos nos idiomas português e inglês, publicados entre 2011 e 2021. Resultados: Evidenciou-se que a presença da equipe multidisciplinar no Programa Saúde da Família proporciona melhor acolhimento, resolução e promoção da saúde nas comunidades em que há equipes interdisciplinares inseridas. Além de promover a integralidade na Atenção Básica e integração com os outros níveis da Rede de Atenção à Saúde. Conclusão: Pode-se perceber que todos os profissionais têm um papel relevante na saúde, sabe-se, ainda que é um processo de trabalho contínuo e a equipe multidisciplinar é fundamental na comunidade para promoção da saúde da população. 
Palavras-chave: Estratégia Saúde da Família; Equipe de Assistência ao Paciente; Atenção. Área Temática: Ciências da Saúde

E-mail do autor principal: angelicalribeiro.ar19@gmail.com

\section{INTRODUÇÃO}

A Declaração de Alma Ata, em 1978, foi um importante marco para o desenvolvimento da Atenção Primária à Saúde (APS), na qual defendia a APS como núcleo central de um sistema de saúde, com contribuições para maior eficiência, efetividade e satisfação do usuário (PAIM, 2012). Nesse sentido, desde os anos 1920 até a hodiernidade, vários modelos de tentativas de organização foram implantados na APS em diferentes regiões do país. Entretanto, o marco mais importante da APS ocorreu com a implantação do Programa Saúde da Família (PAIM, 2012; MENDES, 2012), reconhecido hoje como Estratégia Saúde da Família (ESF) pela sua capacidade em orientar a organização do sistema de saúde (MENDES, 2012).

Desde 1994, o Programa Saúde da Família (PSF) é a principal porta de entrada do sistema de saúde, acolhendo e procurando respostas para a maioria dos problemas de saúde da população, a fim de reduzir danos e sofrimentos, e responsabilizando-se pela efetividade do cuidado, ainda que oferecido em outros pontos da rede. Para isso, se faz necessário o trabalho em equipe de profissionais de áreas distintas, para que seus saberes se somem e tornem o cuidado efetivo na sua população alvo (BRANDÃO; OLIVEIRA, 2016).

Historicamente, o modelo prevalente de formação em saúde é biomédico, focalizado nas intervenções dos sintomas e secundarizando os demais aspectos do processo de saúdedoença dos indivíduos (SHIMIZU, CARVALHO JÚNIOR, 2012). Nesse viés, o PSF tem como objetivo priorizar a promoção da saúde, mudando o foco da atenção à saúde centrada no médico e na doença, rompendo com o modelo tradicional e individualista (OLIVEIRA, 2016).

O PSF é um importante modelo para garantir uma Atenção Primária de Saúde (APS) forte no SUS, aliada a políticas que priorizem os atributos essenciais da APS, sobretudo pela inovação em tecnologias assistenciais, de gestão e de comunicação (TASCA, R., et al., 2020). A integração entre os diferentes profissionais da saúde apresenta fortes potencialidades ao programa, trazendo inúmeros benefícios aos usuários (FILHO, SAMPAIO, BRAGA, 2017). Diante do exposto, este estudo tem como objetivo identificar na literatura científica a importância do trabalho da equipe multidisciplinar no programa saúde da família. 


\section{METODOLOGIA}

O presente estudo é uma revisão integrativa da literatura científica, pois contém uma ampla abordagem com rigor metodológico, combinando dados da literatura empírica e teórica, sintetizando resultados obtidos em pesquisas disponíveis sobre a temática, de forma ordenada e abrangente. (SOUZA, 2010). Possui abordagem quantitativa, de natureza exploratória e descritiva. Foi realizada através da busca online de artigos científicos nacionais e internacionais, no período de setembro de 2021.

Esta revisão foi elaborada a partir das seguintes etapas: escolha do tema, construção da pergunta de pesquisa através do acrônimo PICo (paciente, interesse, contexto), escolha dos Descritores em Ciências da Saúde (DeCS), definição dos critérios de inclusão/exclusão dos artigos científicos; coleta, análise e discussão dos dados dos estudos selecionados, exposição da síntese das evidências encontradas.

A questão norteadora foi definida a partir do PICo. A população estudada foi a equipe multidisciplinar, com interesse na importância do seu trabalho no contexto do Programa de Saúde da Família. Dessa forma, questionou-se qual a importância do trabalho da equipe multidisciplinar no programa saúde da família?

Após esta etapa foi realizada a busca nas bases de dados Scientific Electronic Library Online (SciELO) e na Literatura Latino-Americana e do Caribe em Ciências da Saúde (LILACS), via Biblioteca Virtual em Saúde (BVS), através dos DeCS: "Estratégia Saúde da Família", "Equipe de Assistência ao Paciente" e “Atenção Primária à Saúde”, combinados entre si pelo operador booleano and.

Foram definidos como critérios de inclusão os estudos primários e secundários, que abordaram a temática, publicados na íntegra, no idioma português e inglês, entre 2011 e 2021 e como critérios de exclusão, artigos repetidos nas bases de dados. Foram selecionados 09 trabalhos científicos.

Para a seleção dos artigos, leu-se o título e o resumo dos estudos encontrados, observando os critérios de elegibilidade. Em seguida, realizou-se uma leitura criteriosa de todos os artigos e iniciou-se a coleta dos dados. Para tanto, foi elaborado um quadro contendo os autores, revista, título e principais achados.

Como este estudo é uma revisão integrativa da literatura, não houve a necessidade de submissão ao Comitê de Ética e Pesquisa (CEP), sendo respeitados os aspectos éticos no que se refere à fidelidade às fontes citadas. 


\section{RESULTADOS E DISCUSSÃO}

Foram reunidos 09 artigos com data de publicação entre 2011 e 2021, nos quais evidenciou-se a importância da atuação multiprofissional no Programa Saúde da Família. A presença de profissionais de várias áreas da saúde na atenção primária possibilita uma melhor assistência aos usuários do Sistema Único de Saúde (SUS). Entretanto, a presença de uma equipe interdisciplinar de saúde ainda não é uma realidade comum em todo o território nacional, visto que ainda há pouca é recente implantação de programas de residência multiprofissional na atenção primária de saúde.

Dessa forma, para a obtenção dos resultados, foi realizada a síntese dos dados coletados com o objetivo de explanar a fundamental importância da atuação da equipe interprofissional no serviço de atenção básica.

Quadro I: Características dos estudos sobre a importância do trabalho da equipe multidisciplinar no programa saúde da família. 


\begin{tabular}{|c|c|c|c|}
\hline AUTORES & REVISTA & TítULo & RINCIPAIS ACHADOS \\
\hline $\begin{array}{l}\text { BEZERRA, R. K. C.; } \\
\text { ALVES, A. M. C. V. }\end{array}$ & $\begin{array}{l}\text { Expressão } \\
\text { Católica Saúde }\end{array}$ & $\begin{array}{l}\text { A IMPORTÂNCIA DO TRABALHO DA } \\
\text { EQUIPE MULTIPROFISSIONAL NA } \\
\text { ESTRATÉGIA SAÚDE DA FAMIILIA E } \\
\text { SEUS PRINCIPAIS DESAFIOS }\end{array}$ & $\begin{array}{l}\text { A atuação da equipe multiprofissional proporciona maior humanidade nos } \\
\text { atendimentos, tornando-os menos medicalizados, além de promover } \\
\text { integralidade, equidade, universalidade, resolubilidade, intersetorialidade e } \\
\text { a participação da comunidade nas transformaçães em saúde. O trabalho } \\
\text { em equipe é um dos determinantes para uma boa atuação da equipe } \\
\text { multidisciplinar. Entretanto, a falta de uma boa preparação visando o } \\
\text { trabalho em equipe ainda é realidade nos cursos de graduação. O déficit na } \\
\text { infraestrutura e a falta de recursos necessários para um bom apoio às } \\
\text { famílias é também um dos desafios encontrados pela equipe } \\
\text { multiprofissional. }\end{array}$ \\
\hline $\begin{array}{l}\text { PEREIRA, R. C. A.; } \\
\text { ROVERA, F. J. U.; } \\
\text { ARTMANN, E. }\end{array}$ & & $\begin{array}{l}\text { O TRABALHO MULTIPROFISSIONAL } \\
\text { NA ESTRATÉGIA SAÚDE DA } \\
\text { FAMÍLIA: ESTUDO SOBRE } \\
\text { MODALIDADES DE EQUIPES }\end{array}$ & $\begin{array}{l}\text { A conversação e compartilhamento de experiências e conhecimentos torna } \\
\text { a equipe multiprofissional melhor para a promoção da saúde no território. } \\
\text { A rotatividade da liderança também é fundamental para o estabelecimento } \\
\text { de uma equipe integrada, assim como a interdependência dos trabalhos } \\
\text { realizados por cada membro da equipe torna possivel a realização das } \\
\text { ações de saúde. O trabalho em equipe possibilita maior abrangência das } \\
\text { ações em saúde. }\end{array}$ \\
\hline $\begin{array}{l}\text { ANTUNES, M. D. } \\
\text { et al. }\end{array}$ & $\begin{array}{l}\text { Perspectiva: } \\
\text { Ciência e Saúde }\end{array}$ & $\begin{array}{l}\text { ATUAÇÃO DO FISIOTERAPEUTA NA } \\
\text { ATENÇÃO BÁSICA - ESF E NASF: } \\
\text { UMA REVISÃO DE LITERATURA }\end{array}$ & $\begin{array}{l}\text { O trabalho do fisioterapeuta no NASF converge com os objetivos das outras } \\
\text { especialidades da saúde atuantes no Núcleo de Apoio. O fisioterapeuta } \\
\text { orienta e presta atendimento à comunidade mediante demanda. }\end{array}$ \\
\hline $\begin{array}{ll}\text { FILHO, E. J. S, } \\
\text { SAMPAIO, } \\
\text { BRAGA, L. A. V. }\end{array}$ & $\begin{array}{l}\text { Tempus - Acta } \\
\text { De Saúd } \\
\text { Coletiva }\end{array}$ & 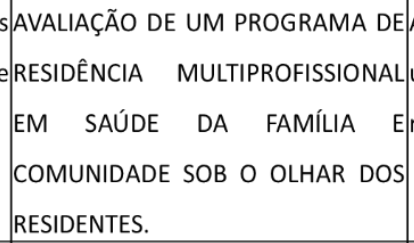 & $\begin{array}{l}\text { A implementação de programas de residência multiprofissional representa } \\
\text { um avanço na formação profissional, apesar de ainda não fazer parte da } \\
\text { realidade de muitas localidades. }\end{array}$ \\
\hline $\begin{array}{l}\text { BRANDÃO, G. C. } \\
\text { G.; OLIVEIRA, M. } \\
\text { A. C. }\end{array}$ & RSC online & 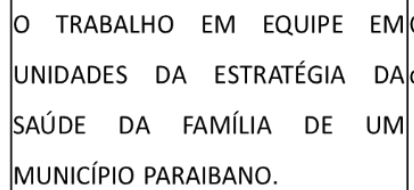 & $\begin{array}{l}\text { O relacionamento da equipe está diretamente relacionado com a qualidade } \\
\text { dos serviços prestados à comunidade. }\end{array}$ \\
\hline
\end{tabular}




\begin{tabular}{|c|c|c|c|}
\hline SILVA, P.A, et al. & $\begin{array}{l}\text { Revista } \\
\text { ConScientia } \\
\text { Saúde }\end{array}$ & $\begin{array}{l}\text { ATUAÇÃO EM EQUIPES } \\
\text { MULTIPROFISSIONAIS DE SAÚDE: } \\
\text { UMA REVISÃO SISTEMÁTICA }\end{array}$ & $\begin{array}{l}\text { A atuação da equipe de multiprofissionais para a saúde pública não só } \\
\text { torna o atendimento mais completo, como oportuniza outros profissionais } \\
\text { de saúde a atuarem na atenção básica de saúde }\end{array}$ \\
\hline $\begin{array}{l}\text { SILVA, C. M. DA; } \\
\text { NASCIMENTO, D. } \\
\text { A.; MAIA, L. F. S. }\end{array}$ & $\begin{array}{l}\text { Revista Remecs: } \\
\text { Revista } \\
\text { Multidisciplinar } \\
\text { de Estudos } \\
\text { Científicos em } \\
\text { Saúde }\end{array}$ & $\begin{array}{l}\text { A ATUAÇÃO DO ENFERMEIRO NA } \\
\text { ESTRATÉGIA } \\
\text { SAÚDE DA FAMÍLIA: COM FOCO EM } \\
\text { PACIENTES } \\
\text { HIPERTENSOS }\end{array}$ & $\begin{array}{l}\text { O papel do enfermeiro como componente da equipe multiprofissional na } \\
\text { ESF é de suma importância, uma vez que juntamente com a equipe a qua } \\
\text { coordena, exerce um contato direto com o paciente e seus familiare } \\
\text { visando a orientação para mudança de estilo de vida e adesão ac } \\
\text { tratamento adequado. }\end{array}$ \\
\hline $\begin{array}{l}\text { LIMA, M. A. M. } \\
\text { de. et al. }\end{array}$ & $\begin{array}{l}\text { Tópicos em } \\
\text { Ciências } \quad \text { da } \\
\text { Saúde, Editora } \\
\text { Poisson. }\end{array}$ & $\begin{array}{l}\text { EFEITOS DA } \\
\text { MULTIPROFISSIONAL NA GARANTIA } \\
\text { DE UM } \\
\text { ENVELHECIMENTO SAUÃO } \\
\text { REVISÃO SISTEMÁTICA }\end{array}$ & $\begin{array}{l}\text { As ações educativas desenvolvidas pela equipe multiprofissional na atenção } \\
\text { primária contribuem para auxiliar em tratamentos de doenças já existentes, } \\
\text { além de reduzir o risco de novas doenças, resultando em uma melhora da } \\
\text { qualidade de vida e um processo de envelhecimento saudável. }\end{array}$ \\
\hline $\begin{array}{l}\text { ARANTES, L. J. et } \\
\text { al. }\end{array}$ & $\begin{array}{l}\text { Ciência \& Saúc } \\
\text { Coletiva }\end{array}$ & $\begin{array}{l}\text { CONTRIBUIÇÕES E DESAFIOS DA } \\
\text { ESTRATÉGIA SAÚDE DA FAMÍLIA } \\
\text { NA ATENÇÃO PRIMÁRIA À SAÚDE } \\
\text { NO BRASIL: } \quad \text { REVISÃO DA } \\
\text { LITERATURA }\end{array}$ & $\begin{array}{l}\text { A adesão a um modelo de equipe multidisciplinar no PSF levou a um } \\
\text { aprimoramento da assistência, devido a interação de diferentes pontos de } \\
\text { vista para o desenvolvimento de ações e ideias, e devido ao enfoque } \\
\text { familiar agrega positivamente a promoção de saúde à comunidade. }\end{array}$ \\
\hline
\end{tabular}

Fonte: Elaborada pelos autores, 2021.

\section{A equipe multiprofissional na garantia da integralidade e qualidade da atenção no Programa de Saúde da Família (PSF)}

Através do estudo de BRANDÃO, G. C. G., é possível destacar a característica essencial do trabalho em equipe em prol da assistência eficiente e eficaz ao indivíduo e a comunidade. A diversidade de campos da saúde proporciona ao território maior qualidade de saúde e faz com que os profissionais tenham contato direto com os determinantes da saúde nas localidades.

Diante disso, a forma de trabalho entre a equipe multiprofissional deve ser realizada de forma articulada e conjunta, uma vez que uma equipe integrada é capaz de desenvolver melhores ações de resolução de problemas de saúde e aprimorar a prática de cuidado, resultando na promoção de uma assistência mais integral que visa melhorar a qualidade de saúde e de vida da comunidade (PEREIRA; RIVERA; ARTMANN, 2013).

Dentre os membros da equipe multiprofissional que atua no Programa Saúde da Família (PSF), cabe citar o papel dos enfermeiros que, segundo estudo publicado por Silva, Nascimento e Maia (2017), necessitam coordenar as equipes de forma integrada como participantes ativos no processo de promoção da saúde, uma vez que os enfermeiros assumem o papel primordial de educadores devido ao relacionamento direto com o paciente e a família, buscando a conscientização do paciente a seguir a forma terapêutica adequada e realizar mudanças em

\section{E - book Tripé do Ensino Superior: Ensino,} Pesquisa e Extensão 
hábitos nocivos, além de serem também responsáveis pelo lado emocional, sempre mantendo um diálogo aberto para que os pacientes desenvolvam confiança e estejam dispostos a compartilhar anseios e dúvidas.

Além disso, o estudo realizado por Antunes et. al. (2020) evidencia o papel do fisioterapeuta no Programa Saúde da Família, que contribui não somente na reabilitação, mas também para a promoção de saúde e prevenção de doenças, por meio da orientação através de palestra que tem como alvo os pacientes, cuidadores e familiares, favorecendo a melhoria na qualidade de saúde e de vida da população.

Apesar dos métodos tradicionais de promoção de saúde, a atuação de uma equipe multiprofissional na atenção primária trás abordagens alternativas de cuidado para com a comunidade, como é demonstrado no estudo de Lima et. al. (2019) que evidencia que a realização de tratamentos não medicamentoso se mostrou eficaz na prevenção de novas doenças, como dinâmicas em grupo, debates sobre entendimento de saúde e experiências de vida, possibilita tomar conhecimento das necessidades de cada grupo e assim facilita a orientação para adotar melhores hábitos de vida.

\section{Residência Multiprofissional no Programa Saúde da Família}

Por meio da avaliação de FILHO, E. J. S., é possível destacar as dificuldades acerca da residência multiprofissional no PSF do estado da Paraíba, por ser uma modalidade ainda recente e escassa, a atuação do profissional residente enfrenta pouco conhecimento não só por parte da população como também pela própria equipe, a qual ainda não encontra-se familiarizada com a atuação de residentes na Atenção Básica.

\section{Desafios enfrentados pela equipe multiprofissional no Programa Saúde da Família (PSF)}

De acordo com o estudo realizado por Silva et. al. (2013), uma das principais razões para haver dificuldades de trabalhar em uma equipe multiprofissional está atrelada à formação acadêmica dos profissionais da saúde, uma vez que as alterações provocadas desde a criação do SUS e a posterior implantação do PSF modificaram o modelo hospitalocêntrico que era centrado na figura do médico, demandando a partir de então um trabalho conjunto dos profissionais das diversas áreas da saúde. Entretanto, o modelo universitário tradicional permanece voltado para a individualidade da profissão, resultando na dificuldade de trabalhar em equipe e em fornecer um atendimento humanizado e de qualidade aos pacientes. 
Além disso, também existem adversidades no cotidiano de trabalho dessas equipes devido à falta de infraestrutura e apoio de gestores, ausência de planejamento de ações, recursos de trabalho insuficientes e inadequados que levam a um desgaste físico e mental dos profissionais (BEZERRA; ALVES, 2019).

Outro desafio presente na atuação da equipe multiprofissional no Programa Saúde da Família (PSF) é a formação de longas filas de espera que, segundo Arantes et. al, (2016), é resultado da carência de serviços especializados para suprir a demanda da comunidade, fator que está relacionado à uma ampliação da atenção primária por meio do PSF mas sem uma expansão simultânea dos profissionais especializados, não atendendo adequadamente à demanda da comunidade.

\section{CONCLUSÃO}

Diante o exposto, circunstancia-se que o indivíduo em questão no que tange a qualidade de vida, e conforme o ideal de integralidade apontado pelo Sistema Único de Saúde (SUS), é pertinente fazer a análise de forma conjunta e integral em prol do bem-estar. A saúde é um processo contínuo e cada profissional tem papel relevante tendo como base a formação exercida.

Enfatizar o autocuidado e um bom atendimento da atenção básica é um aspecto essencial, tendo em vista que é justamente nesse processo que a população busca exercer atividades que estabilizam o quadro clínico de saúde. Sendo assim, é imprescindível ampliar ainda mais essa área, a participação de demais profissionais para fazer parte da composição multiprofissionais é algo preciso, e os maiores privilegiados com esse cenário é a comunidade. 


\section{REFERÊNCIAS BIBLIOGRÁFICAS}

BEZERRA, R.K.C.; ALVES, A.M.C.V. A importância do trabalho da equipe multiprofissional na Estratégia Saúde da Família e seus principais desafios. Revista Expressão Católica Saúde, [S.1.], v. 4, n. 2, p. 7-15, dec. 2019. ISSN 2526-964X.

PEREIRA, R.C.A.; RIVERA, F.J.U.; ARTMANN, E. O trabalho multiprofissional na estratégia saúde da família: estudo sobre modalidades de equipes. Interface - Comunicação, Saúde, Educação [online], v. 17, n. 45, pp. 327-340, 2013.

ANTUNES, M. D. et al. Atuação do fisioterapeuta na AtençãoBásica - ESF e NASF: Uma revisão de literatura. Revista Perspectiva: Ciência e Saúde, Osório, V. 5 (2): 86-100, Ago 2020.

JOSÉ DOS SANTOS FILHO, E.; SAMPAIO, J.; BRAGA, L. A. V. A avaliação de um programa de residência multiprofissional em Saúde da Família e a comunidade sob o olhar dos residentes. Tempus - Actas de Saúde Coletiva, v. 10, n. 4, p. Pág. 129-149, 14 jul. 2017.

BRANDÃO, G.C.G; OLIVEIRA, M.A.C. O Trabalho em equipe em UBS (PB). RSC online, 2016; 5(3): p.51-62.

SILVA, P.A et al. Atuação em equipes multiprofissionais de saúde: uma revisão sistemática. Revista ConScientia e Saúde, v. 12, n. 1, p. 153-160, 2013.

SILVA, C. M. DA; NASCIMENTO, D. A.; MAIA, L. F. S. A atuação do enfermeiro na estratégia saúde da família: com foco em pacientes hipertensos. Revista Remecs: Revista Multidisciplinar de Estudos Científicos em Saúde, v. 2, n. 3, p. 7-17, 2017.

LIMA, M. A. M. de. et al. Efeitos da atuação multiprofissional na garantia de um envelhecimento saudável: revisão sistemática. Tópicos em Ciências da Saúde, Editora Poisson, v. 8, ed.1, 2019.

ARANTES, L. J. et al. Contribuições e desafios da estratégia saúde da família na atenção primária à saúde no Brasil: revisão da literatura. Ciência \& Saúde Coletiva, v. 5, maio, 2016. 
MENDES, E.V. A APS no Brasil. O cuidado das condições crônicas na Atenção Primária à Saúde: o imperativo da consolidação da Estratégia da Saúde da Família. Brasília: Organização Pan-Americana da Saúde; 2012. p. 71-137.

OLIVEIRA, C. F.; ARRUDA, G. M. M. S.; MELO, A. L. A., BARCELOS, S. C. Cuidado interprofissional aos portadores de Hipertensão e Diabetes em um grupo de intervenção educacional e terapêutica. Cadernos ESP. Ceará. v. 10, n. 2, p. 33-45, 2016.

PAIM, J. S. Atenção Primária à Saúde: uma receita para todas as estações? Saúde Debate 2012; 36(94):343-347.

SHIMIZU, H. E.; CARVALHO JUNIOR, D. A. C. O processo de trabalho na Estratégia Saúde da Família e suas repercussões no processo saúde-doença. Ciência \& Saúde Coletiva. v. 17, n. 9, p. 2405-2414, 2012.

TASCA, R., et al. Recomendações para o fortalecimento da atenção primária à saúde no Brasil. Rev Panam Salud Publica, v, 44, ed.4, 2020. 